\title{
Resilience and sustainability
}

\author{
Resilience is important, but context and details matter.
}

M ost sustainability scholars and practitioners adapt their explicit or implicit definition of resilience from its roots in ecology. C. S. Holling introduced the concept as an alternative to the more engineering-oriented, quantitative measure that was often used to assess the rate at which a system returns to equilibrium following disturbance ${ }^{1,2}$. This view typically assumed that a system has equilibrium conditions and a single state to which that equilibrium applies. In contrast, many ecological and social-ecological systems may spend more time out of equilibrium and in a number of possible ('alternative stable') states. A more flexible conceptualization such as this recognizes the complexity of these systems and the often changing nature of their context. Indeed, keeping context in mind is vital for considering the subtle dance between resilience and sustainability.

Despite decades of work and hundreds of papers discussing resilience and sustainability, some broadly and others in terms of specific systems and issues, the two words are still often used interchangably. In some ways this is understandable, as sustainability scholarship, much like other areas of research, is awash with terminology and buzzwords that provide valuable shorthand. Carefully defining all such terms would expand papers considerably, introducing a difficult balancing act in this era of word limits.

In this issue of Nature Sustainability, three papers consider the relationship between resilience and sustainability. In an Article, Grêt-Regamey et al. focus on how social-ecological diversity in Swiss mountain farming communities moderates their resilience in the face of environmental change and the level of sustainability that this implies. Tu and colleagues' Article focuses on the network structure linking natural resources and end users, theoretically exploring how the food trade network can affect the sustainability of the natural resources on which it depends. Finally, in a Perspective, Elmqvist et al. compare the resilience and sustainability of urban systems transforming in response to global change.

Resilience can be considered in terms of structure, function or a combination of both dimensions. Grêt-Regamey et al. focus on a farming system's ability to absorb shocks and reorganize given climatic and socioeconomic changes, in order to continue the function of producing ecosystem services.

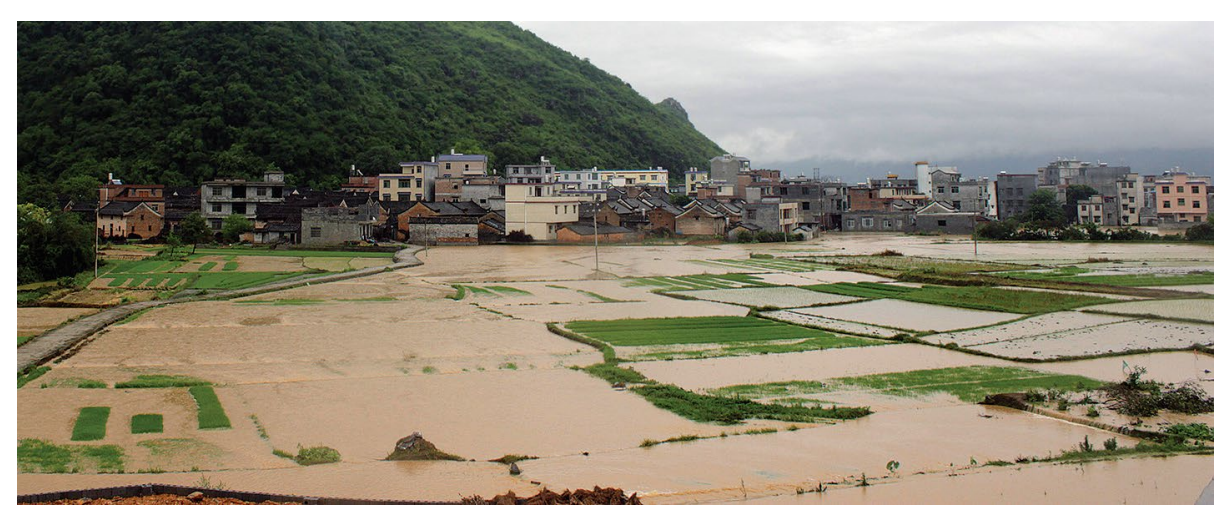

Farmland is flooded in Gepo Township, Hezhou City, south China's Guangxi Zhuang Autonomous Region, 11 May 2015. Credit: Xinhua/Alamy Live News

Tu et al. measure resilience as the probability that the global food system in their model exists in a sustainable state, where the associated natural resources are not degraded or depleted. They focus on how resilient this 'sustainable state' is to different network arrangements and therefore examine the structure of their system. Finally, Elmqvist et al. note that cities are strikingly resilient, with many lasting for millennia in the face of remarkable environmental, social, cultural and economic changes. Their structure has continued to sustain a vital function, organizing the settlement and employment arrangements for our prosocial, hypercultural species. The authors implore us to consider context even more broadly: resilience from what and to what? What is being sustained in the process?

Cities tend to persist in the face of natural disasters, but by concentrating people and infrastructure they sustain unusually high costs, as evidenced by the effects of recent hurricanes, tsunamis and floods on cities, coasts and floodplains. Mountain farming systems may persist, but at the occasional expense of individual farmers who, like urban business owners, may have to sell to others whose management practices better align with current conditions. The global food system is obviously adapting and persisting, but examining its structure over time suggests that its increasing connectivity and decreasing modularity - the degree to which a network is composed of distinct subnetworks - is actually eroding its resilience.

Whether focusing on a socioenvironmental system's structure or its function, resilience informs sustainability, but the context remains important. Is a farming community, food system or city that is resilient to climate shocks sustainable? What if those shocks - as explored in Meyer and colleagues' Article in November's issue - arrive at increasing frequency? What if these systems are tethered to others, such as farmlands or fisheries, which are not themselves so resilient?

These realities, inherent to all complex adaptive systems, emphasize the importance of defining resilience and sustainability relative to the system boundaries, ideally ones large enough to capture reasonable feedback effects. They also demonstrate the importance of taking a long-term view or, at the very least, considering time explicitly. Of course, no study can assess or 'do' it all, but it is only by considering a multitude of different factors that we can deepen our understanding of sustaining the evolving human enterprise on our productive but fragile and finite planet. An important goal of Nature Sustainability is to feature research and opinion that illuminates these connections and realities - explorations that, when considered together, will deepen our understanding of the relationship between resilience and sustainability, and the potential ways that they are linked.

Published online: 9 April 2019

https://doi.org/10.1038/s41893-019-0284-4

\footnotetext{
References

1. Folke, C. et al. Ecol. Soc. 15, 20 (2010).

2. Holling, C. S. Annu. Rev. Ecol. Syst. 4, 1-23 (1973).
} 